\title{
Open thoracic anterolateral cordotomy for pain relief in children: report of 2 cases
}

\author{
Dora Steel, MRCPCH, Matthew A. Kirkman, MRCS, MEd, Dominic N. P. Thompson, FRCS(SN), and \\ Kristian Aquilina, MD, FRCS(SN)
}

Department of Neurosurgery, Great Ormond Street Hospital, London, United Kingdom

\begin{abstract}
Open anterolateral cordotomy is an effective treatment option for adults with intractable pain, but it has seldom been attempted in children. In the 2 previously reported cases in children, the procedure was used within 10 days of death from neoplastic disease. In this paper the authors describe 2 cases in which open cordotomy was used successfully in children outside the immediate terminal phase of disease. Both patients experienced effective analgesia with minimal adverse effects. The authors propose that consideration of cordotomy as an option for the management of intractable pain in children does not need to be delayed until death is imminent.
\end{abstract}

https://thejns.org/doi/abs/10.3171/2017.5.PEDS17119

KEY WORDS intractable pain; spinothalamic tract; thoracic cordotomy; surgical technique; spine

$\mathrm{F}$ IRST performed in 1912, open anterolateral thoracic cordotomy is an effective option for adults with intractable pain, but it has seldom been attempted in children. The development of implantable pumps and spinal cord stimulators, as well as the increased use of bilateral percutaneous cervical cordotomy, have reduced the relevance of open thoracic anterolateral cordotomy in the management of intractable pain. ${ }^{17}$ This procedure still has an important role in select situations. Division of the anterolateral white matter of the thoracic spinal cord disrupts the lateral and anterior spinothalamic tracts and is effective at abolishing pain arising from the contralateral lower limb, lower abdomen, and pelvis.

Only 2 cases of pediatric open anterolateral cordotomy have been described; both cases involved children with terminal neoplastic disease who underwent the procedure within the last 10 days of life. ${ }^{13,14}$ In this paper we describe 2 cases in which open cordotomy was used successfully in children outside the immediate terminal phase. Both children experienced effective analgesia with minimal adverse effects. We propose that consideration of cordotomy as an option for the management of intractable pain does not need to be delayed until the death of the child is imminent.

\section{Anatomy and Surgical Technique}

Pain signals are carried from the periphery through unmyelinated $\mathrm{C}$ and small myelinated delta $\mathrm{A}$ nerve fibers to the dorsal root entry zone. They enter the dorsolateral tract of Lissauer, ascend or descend several spinal levels, and then enter Laminae I-V of the dorsal horn. A segment's principal input to the dorsal horn generally occurs approximately 3 segments rostral to its dorsal root entry zone. At the dorsal horn, the axons of the first-order neurons synapse with marginal cells or wide dynamic range neurons. These second-order neurons decussate in the anterior white commissure and ascend in the contralateral lateral spinothalamic tract. Fibers are arranged somatotopically within the tract, with the sacral fibers posterolateral and the cervical fibers anteromedial. The corticospinal tract, running within the lateral funiculus of the spinal cord, lies dorsal to the lateral spinothalamic tract. The dentate ligament at the surface of the spinal cord is an important landmark that demonstrates the point of separation between the two tracts. $^{10}$

Under general anesthesia, the patient is positioned prone and the T-3 spinous process is identified on lateral plain radiography. The dura is typically exposed through a 
T3-5 laminoplasty and opened through a paramedian longitudinal incision. The durotomy is extended laterally in a "D" shape to allow a flap to be retracted contralateral to the side of the cordotomy. The ipsilateral dentate ligament is divided at its dural insertion and used to rotate the cord to the contralateral side. The point midway between the insertion of the dorsal and ventral rootlets marks the position of the sacral fibers within the lateral spinothalamic tract and forms the posterior limit of the cordotomy. Rostral fibers run anteromedial to the sacral fibers within the spinothalamic tract in a somatotopic fashion. The lateral corticospinal tract is situated posterior to this point and should be safe from injury.

A no. 11 blade is broken off at a length of $4.5 \mathrm{~mm}$ and held in an artery forceps. Through an avascular segment the pia is first divided using a knife or microscissors, starting from a point midway between the ventral and dorsal rootlets. This prevents distortion of the rotated cord as the incision proceeds. The blade is then inserted to a depth of $4.5 \mathrm{~mm}$ at an angle of $15^{\circ}$ degrees anteriorly (Fig. 1). The cord is divided using a circular movement from deep to superficial. The anteromedial limit of the incision is $2.5 \mathrm{~mm}$ anterior to the ventral rootlets.? The anterior spinal artery lies just beyond this anterior limit at $4.5 \mathrm{~mm}$ anteromedial to the center of the ventral rootlet (Fig. 2) ${ }^{8}$ For bilateral cordotomy the procedure is repeated on the contralateral side, at least 1 spinal segment below the first. The dura is then closed in a watertight fashion, and the lamina is replaced.

\section{Case Reports \\ Case 1}

History and Examination

An 11-year-old boy with neurofibromatosis Type I and a large, inoperable, malignant nerve sheath tumor of the right sciatic nerve, with extensive pelvic involvement, underwent several cycles of daunorubicin and trabectedin chemotherapy without significant oncological benefit (Fig. $3)$. He developed severe pain in the right hip and buttock with limited response to high-dose conventional analgesics, including gabapentin and morphine. He underwent implantation of an intrathecal infusion pump, which allowed continuous delivery of morphine and bupivacaine. Increasing doses of intrathecal analgesics led to pain control for 2 months. Unfortunately, his requirement for additional intravenous oxycodone analgesia increased as the lesion ulcerated through the skin. Although initially predominantly neuropathic, his pain became harder to control as progressive tumor growth within the pelvis and gluteal region led to dislocation of the right hip. Despite maximal intrathecal analgesic treatment, his pain was exacerbated by even slight movement and by this stage he was unable to tolerate basic nursing care. On two occasions he required general anesthesia for transfers between his bed and a hospital trolley.

\section{Operation and Postoperative Course}

Three months after implantation of the pump, and following discussion with his family, a bilateral open cordotomy at the T-5 and T-6 levels, via T3-6 laminoplasty,
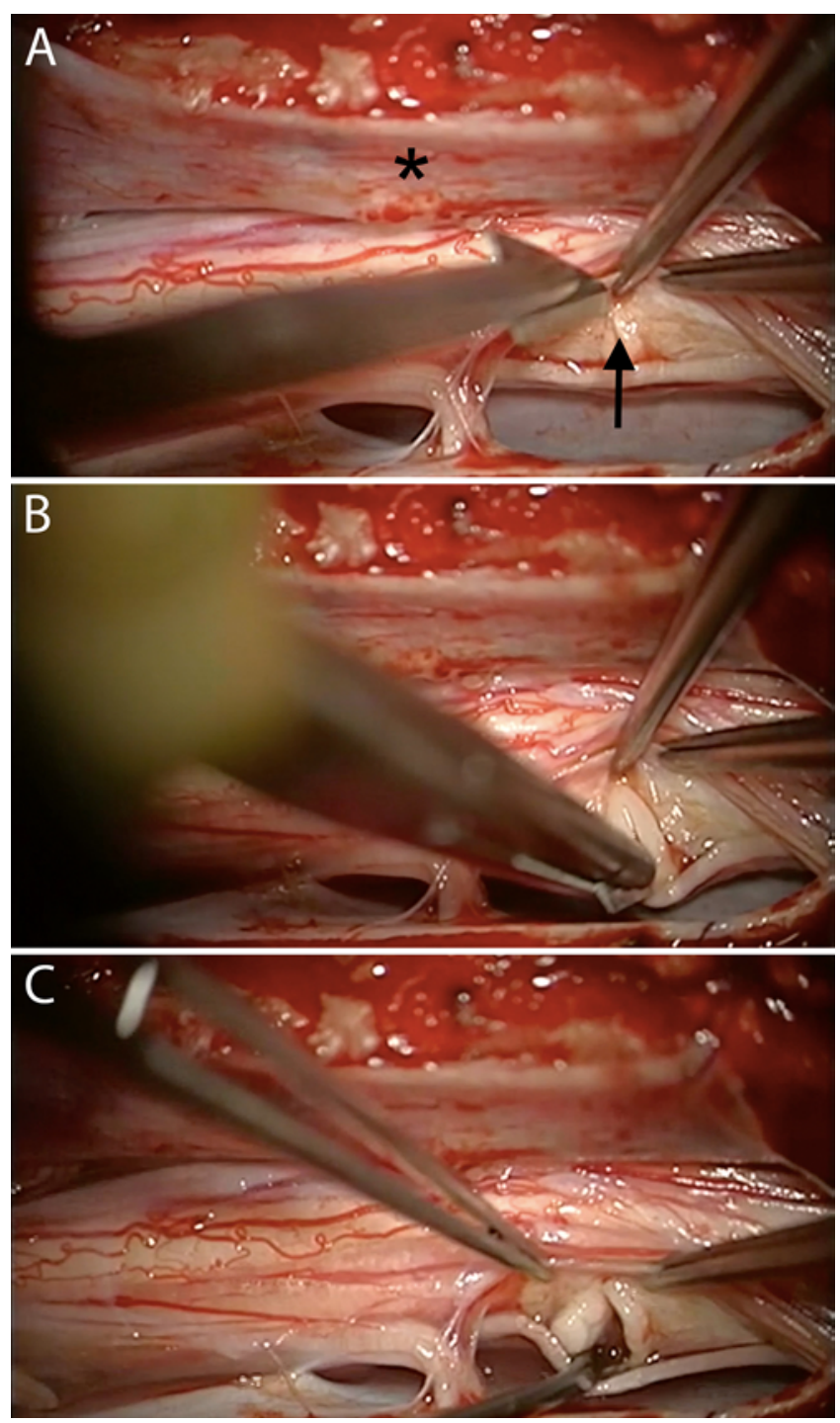

FIG. 1. Images from the intraoperative microscope during Case 1. A: From a posterior approach, and following creation of a D-shaped dural flap (asterisk), the denticulate ligament is divided and held with forceps to gently rotate the spinal cord, revealing the anterolateral aspect of the side of the spinal cord undergoing cordotomy (arrow). A knife blade is then inserted into the lateral aspect of the spinal cord with the blade directed toward the anterior spinal cord. B: The anterolateral spinal cord is divided using a circular movement from deep to superficial. C: The extent of the anterolateral cordotomy can be confirmed with a blunt surgical instrument. Figure is available in color online only.

was performed. A bilateral procedure was undertaken as it appeared likely that tumor spread would lead to left-sided pain in the near future. Postoperatively there was immediate and significant improvement in his pain. There was no reduction in lower-limb motor function. He was able to turn in bed and sit up and was discharged home 2 weeks after the procedure on low-dose oral morphine. His intrathecal analgesic treatment was successfully discontinued. As expected, the adverse effects of his analgesics, and specifically his intractable constipation, also improved.

By 8 weeks, despite progressive tumor growth, his opiate analgesia was discontinued. He remained pain free. 


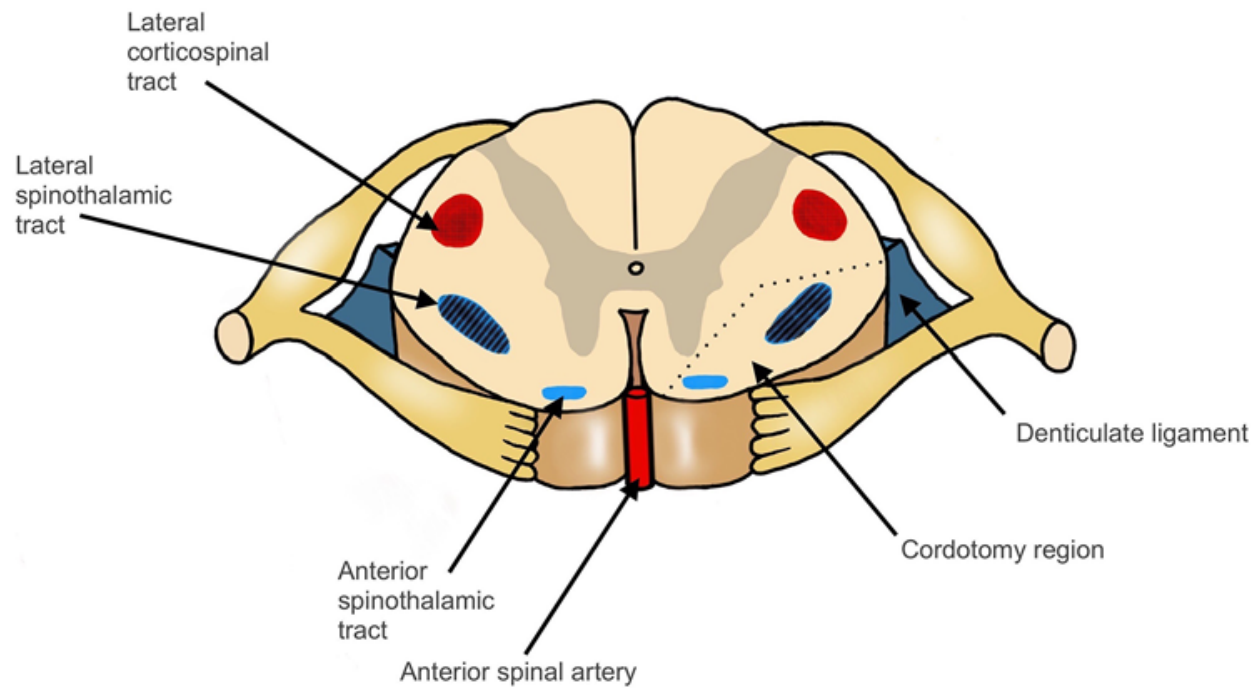

FIG. 2. Illustration of a segment of the thoracic spinal cord, showing the region that is incised during the anterolateral cordotomy, which lies between the denticulate ligament laterally and the anterior spinal artery anteromedially. The cordotomy incorporates the lateral and anterior spinothalamic tracts. Copyright Kristian Aquilina. Published with permission. Figure is available in color online only.

He was able to interact comfortably with his family for a further 4 weeks until he developed severe shoulder pain related to new metastatic disease. This pain was successfully managed with oral oxycodone and fentanyl patches. There was no pain distal to his lower abdomen and pelvis. He remained at home, where he died 12 weeks after the anterolateral cordotomy.

\section{Case 2}

History and Examination

A 10-year-old boy was under regular care for caudal regression syndrome that involved sacral agenesis, lumbar dysraphism with a large lipoma and meningocele, bilateral hip dislocation, and exomphalos (Fig. 4). The lipoma,
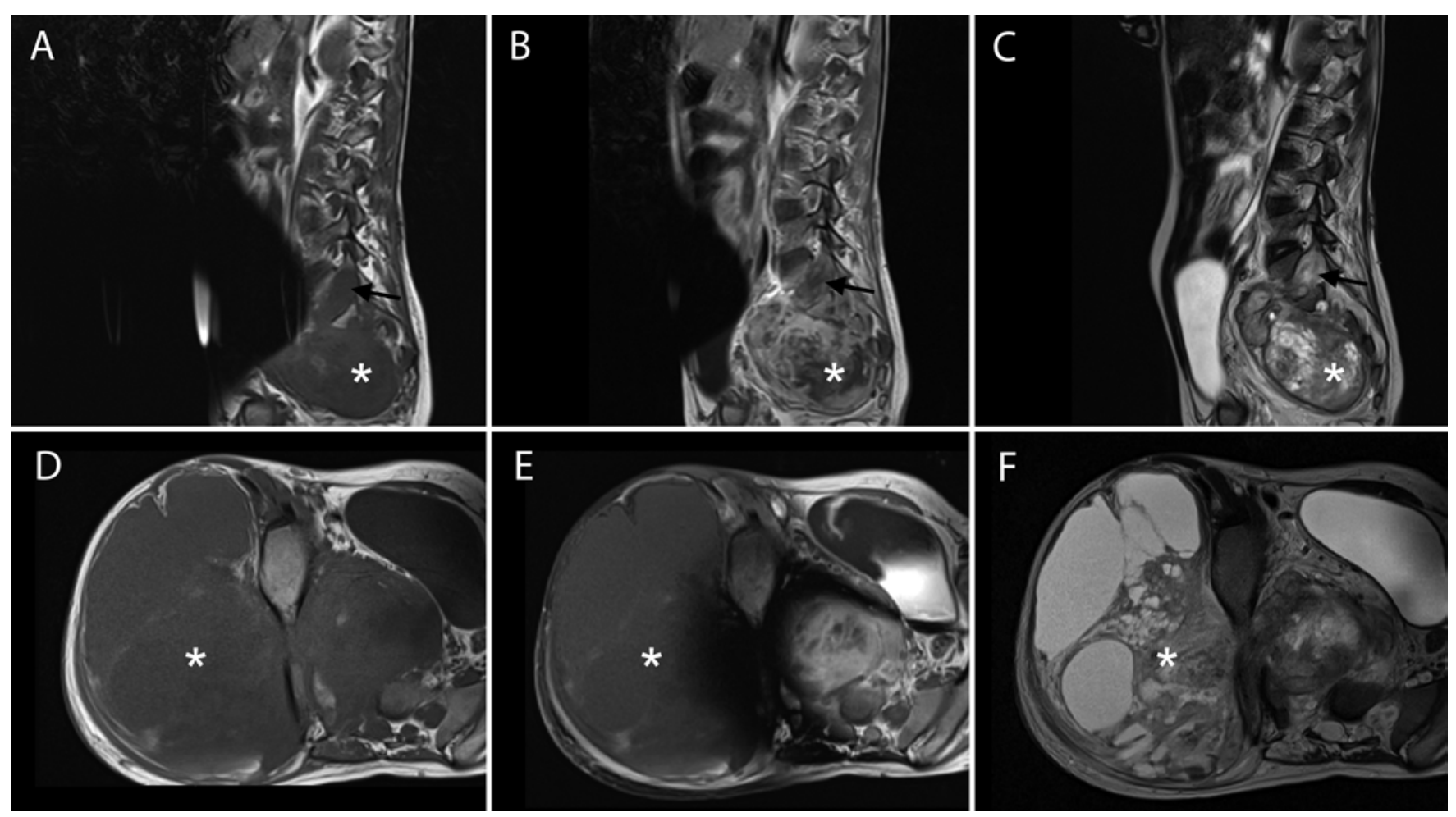

FIG. 3. Preoperative imaging from Case 1. Sagittal and axial T1-weighted precontrast ( $A$ and $D), T 1$-weighted postcontrast (B and $\mathrm{E}$ ), and T2-weighted ( $\mathrm{C}$ and $\mathbf{F}$ ) MRI slices demonstrating a large malignant nerve sheath tumor (asterisks) arising in relation to the right L-5 (arrows) and all sacral nerve roots. The tumor traverses the right sciatic notch and occupies the right gluteal region. The mass demonstrates peripheral cystic degeneration. 

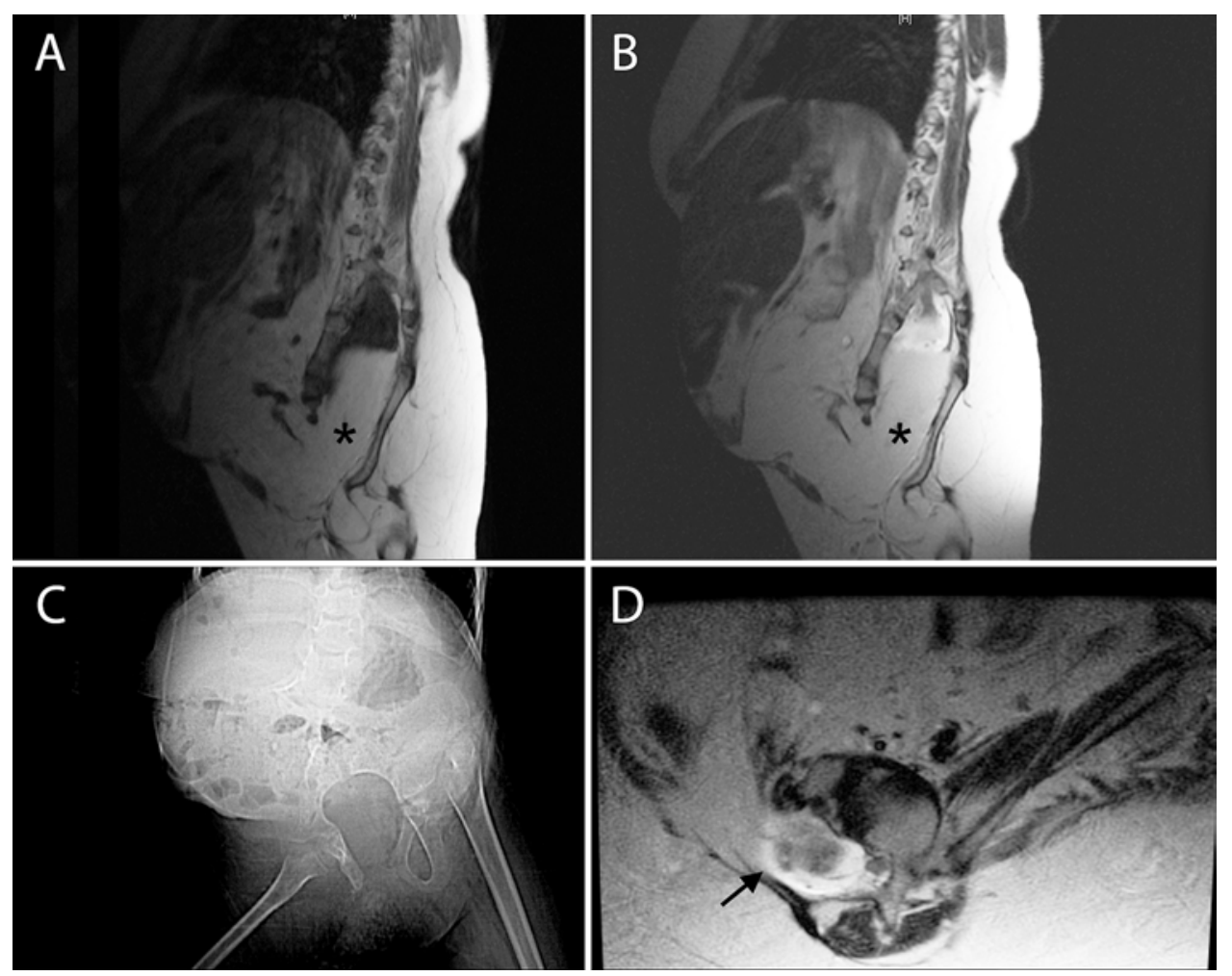

FIG. 4. Preoperative imaging from Case 2. Sagittal T1-weighted (A), sagittal T2-weighted (B), and axial T2-weighted (D) MRI slices demonstrating the presence of sacral dysgenesis and associated dysraphism. There is a large, right, lateral lumbar lipomeningocele (arrow and asterisk). The anteroposterior scout CT image (C) illustrates the dysplastic pelvis and partial sacral agenesis.

which extended into the pelvis and subcutaneous tissues of the gluteal region, caused progressive deep abdominal and pelvic pain. He had required a colostomy as well as an ileal conduit for bladder drainage in infancy. He had paraplegia, with only mild left hip flexion evident on examination.

Increasing nociceptive pain related to the right pelvic and gluteal mass necessitated progressive escalation of analgesic medication over 4 years, with increasing doses of morphine, gabapentin, and fentanyl. Despite maximal doses and significant adverse effects, his pain consistently interfered with mobility and sleep. His poor mobility led to secondary left hip pain and early skin ulceration in the right gluteal region. In view of the size of the lipoma, the condition of the overlying skin, and the neuropathic nature of the gluteal pain, resection of the mass was associated with a high risk of complications and was not expected to lead to a reduction in pain. In addition, an implanted intrathecal pump or spinal cord stimulator would have been difficult to maintain within the distant community where he lived. Moreover, the pain in his deep abdomen, groin and lower extremities was unlikely to be covered well with a spinal cord stimulator.

\section{Operation and Postoperative Course}

Despite uncertainty of the long-term effectiveness of the procedure, the patient underwent a left anterolateral cordotomy at T-5 after careful counseling. The procedure was uncomplicated and left hip flexion was maintained. There was an immediate reduction in his gluteal pain, allowing weaning of his morphine, gabapentin, and fentanyl. His mobility also improved, with resolution of his secondary hip pain. He was discharged home 1 week after the procedure, with significant improvement in his quality of life. On review 36 months postoperatively, analgesic medications have been discontinued and he is now attending school.

\section{Discussion}

Open anterolateral cordotomy involves surgical division of the anterolateral column, including the spinothalamic tract, for relief of intractable pain. This procedure was first performed in 1912.,17 The percutaneous cervical approach, where the spinothalamic pathways were interrupted using a Strontium-90 needle in the upper cervical cord, was introduced in the 1960s and rapidly became the preferred technique. ${ }^{11}$ In a subsequent modification, the needle was replaced by a radiofrequency thermal lesion. ${ }^{15}$

Open cordotomy interrupts all the pain afferents below the level of the lesion and is effective for analgesia from 4 to 5 levels caudally on the contralateral side. ${ }^{10}$ Although cordotomy is not suitable for isolated upper-limb and shoulder pain, where analgesia would be associated with a numb trunk and lower limb, when performed at a midthoracic level it is ideal for lower-limb and pelvic pain. ${ }^{6,10}$

The development of reliable intrathecal analgesic pumps and spinal cord stimulators in the 1990s led to a general reduction in the use of cordotomy procedures. In addition, concerns related to the adverse effects were 
significant, primarily secondary dysesthesia, urinary retention and, for bilateral cervical cordotomy, sleep apnea due to division of the reticulospinal tracts ("Ondine's curse"). $6,16,18$

Neuroablative procedures for pain remain useful in situations in which chronic intrathecal therapy is ineffective or impractical. Cordotomy is performed once, and patients do not need to return for surveillance of their pain control. Unlike intrathecal therapy, no regular commitment to refilling or battery change is required; this is particularly important in communities where such services are not available. Analgesics can be successfully weaned following an effective procedure, resulting in a reduction of the adverse effects typical of chronic opiate use.

Currently most cordotomies are undertaken on adults with pain below the neck related to malignant disease, and are almost always performed through the $\mathrm{C} 1-2$ percutaneous route. ${ }^{1,2,5,9,19}$ In this procedure, the patient is positioned prone; the patient's cooperation is required during stimulation of the target as the typical pain is reproduced. It is therefore unsuitable for most children. Using CT guidance and preprocedural injection of contrast medium through lumbar puncture, a 20-gauge needle is passed ventral to the dentate ligament. Its $2-\mathrm{mm}$ exposed tip delivers the radiofrequency lesion and monitors temperature. Penetration of the spinal cord is associated with a rise in impedance and transient intense neck pain. Prior to lesioning, stimulation is performed to ensure that the needle is in a correct position. Low-intensity and low-frequency stimulation elicits a motor response, which, in the correct position, is evident in the trapezius. At higher intensity and frequency, sensory stimulation occurs, described as contralateral tingling, warmth, cold, or pain. The correct position of the needle within the somatotopically organized spinothalamic tract should cause sensory changes in the region of pain. ${ }^{9} \mathrm{~A}$ recent meta-analysis has evaluated published results for percutaneous C1-2 anterolateral cordotomy in adults for cancer pain and concludes that this procedure should be considered before systematic use of strong opioids. ${ }^{2}$

In a single reported pediatric case involving a 9-yearold boy with refractory neoplastic right lower-limb pain, a percutaneous CT-guided C1-2 cordotomy was effective for pain control. ${ }^{14}$ Intermittent propofol boluses were used to ensure cooperation at critical moments. The child died 10 days postoperatively.

Because most percutaneous cordotomy procedures are performed for malignant pain, long-term follow up is rare. Although more than half of the patients typically experience satisfactory pain relief immediately after the procedure, the pain may recur within 6-12 months in as many as $40 \%{ }^{4,12}$ It is not clear whether this may be related to progression of the tumor, rather than failure of the lesion., ${ }^{4,10}$ One case report, describing an adult undergoing cervical percutaneous cordotomy for intractable posttraumatic lumbosacral pain, was reported to remain pain free for 40 years. ${ }^{3}$ In 22 studies, complete pain relief ranged from $63 \%$ to $90 \%$, while significant pain relief ranged from $59 \%$ to $96 \%{ }^{3}$ In patients with nonmalignant conditions, the success rate in 1 series was reported at $20 \%$ at 3 years. ${ }^{4}$ Rosomoff et al. found satisfactory pain relief in $90 \%$ of patients immediately after the procedure, $84 \%$ at 3 months, $61 \%$ at
1 year, $43 \%$ after $1-5$ years, and $37 \%$ after $5-10$ years. ${ }^{15}$ The mechanism through which pain sensation may return is unknown, and there has been no documented regeneration of the spinothalamic tract. In 1 patient, pain returned in a monotonic rostral-to-caudal fashion, suggesting a dorsolateral progression of spinothalamic tract recovery. ${ }^{3}$ An early report on anterolateral cordotomy for nonmalignant conditions reported on 13 adults, ${ }^{4}$ in which $85 \%$ obtained initial relief, but the success rate fell to $35 \%$ at 1 year and $20 \%$ at 3 years of follow-up.

Percutaneous cordotomy at $\mathrm{C} 1-2$ carries a risk of injury to the descending fibers of the lateral reticulospinal tract that mediate involuntary respiratory drive; these are situated medial to the anteromedial spinothalamic tract, capping the ventral horn. Bilateral $\mathrm{Cl}-2$ cordotomies need to be staged in 2 procedures for this reason. In the patient series of Cowie and Hitchcock, 2 of 56 patients died of respiratory failure. ${ }^{4}$ Open thoracic cordotomy, in view of its level, carries a lower risk of respiratory deterioration, and it is possible to undertake bilateral cordotomy in the same procedure. It is still recommended, however, that bilateral cordotomy be performed across at least 1 or 2 segments, with 1 side divided below the other; this minimizes the impact of potential injury to the lateral reticulospinal tract. Similarly, the risk to urinary incontinence is lower as at least some of the micturition fibers that lie medial to the lateral spinothalamic tract are preserved. It is, therefore, appropriate for patients with preexisting respiratory dysfunction or bilateral pain. In children, it ensures effective bilateral pain relief in a single procedure.

The most common complications of open anterolateral cordotomy are urinary retention and incontinence. ${ }^{10,16}$ Permanent dysesthesia and hemiparesis have also been reported. ${ }^{6}$ In both our patients, the futility of alternative treatment modalities and the severe disability already incurred implied that the impact of complications related to the procedure were less relevant. Lower-limb movements were maintained postoperatively in both patients. In summary, open anterolateral cordotomy is a useful and effective procedure to improve pain control in selected children. Its immediate analgesic effect can promptly and significantly improve quality of life in this patient population. Careful patient selection minimizes the impact of the potential adverse events associated with this procedure. Although its long-term benefit in children remains unclear, it represents a useful option to control pain when all other modalities have been unsuccessful.

\section{References}

1. Bain E, Hugel H, Sharma M: Percutaneous cervical cordotomy for the management of pain from cancer: a prospective review of 45 cases. J Palliat Med 16:901-907, 2013

2. Bellini M, Barbieri M: Percutaneous cervical cordotomy in cancer pain. Anaesthesiol Intensive Ther 48:197-200, 2016

3. Collins KL, Taren JA, Patil PG: Four-decade maintenance of analgesia with percutaneous cordotomy. Stereotact Funct Neurosurg 90:266-272, 2012

4. Cowie RA, Hitchcock ER: The late results of antero-lateral cordotomy for pain relief. Acta Neurochir (Wien) 64:39-50, 1982

5. Jackson MB, Pounder D, Price C, Matthews AW, Neville E: Percutaneous cervical cordotomy for the control of pain in 
patients with pleural mesothelioma. Thorax 54:238-241, 1999

6. Jones B, Finlay I, Ray A, Simpson B: Is there still a role for open cordotomy in cancer pain management? J Pain Symptom Manage 25:179-184, 2003

7. Kahn EA, Peet MM: The technique of anterolateral cordotomy. J Neurosurg 5:276-283, 1948

8. Kahn EA, Rand RW: On the anatomy of anterolateral cordotomy. J Neurosurg 9:611-619, 1952

9. Kanpolat Y, Ugur HC, Ayten M, Elhan AH: Computed tomography-guided percutaneous cordotomy for intractable pain in malignancy. Neurosurgery 64 (3 Suppl):ons187ons194, 2009

10. Konrad P: Dorsal root entry zone lesion, midline myelotomy and anterolateral cordotomy. Neurosurg Clin N Am 25:699 722,2014

11. Mullan S, Harper PV, Hekmatpanah J, Torres H, Dobbin G: Percutaneous interruption of spinal-pain tracts by means of a Strontium ${ }^{90}$ needle. J Neurosurg 20:931-939, 1963

12. Piscol K: [Open spinal operations (anterolateral chordotomy and commissural myelotomy) in modern treatment of pain (author's transl).] Langenbecks Arch Chir 342:91-99, 1976 (Ger)

13. Queinnec MC, Estève M, Vedrenne J: Positive effect of regional analgesia (RA) in terminal stage paediatric chondrosarcoma: a case report and the review of the literature. Pain 83:383-385, 1999

14. Reddy GD, Okhuysen-Cawley R, Harsh V, Viswanathan A: Percutaneous CT-guided cordotomy for the treatment of pediatric cancer pain. J Neurosurg Pediatr 12:93-96, 2013

15. Rosomoff HL, Sheptak P, Carroll F: Modern pain relief: percutaneous chordotomy. JAMA 196:482-486, 1966

16. Sanders M, Zuurmond W: Safety of unilateral and bilateral percutaneous cervical cordotomy in 80 terminally ill cancer patients. J Clin Oncol 13:1509-1512, 1995

17. Spiller WG, Martin E: The treatment of persistent pain of organic origin in the lower part of the body by division of the anterolateral column of the spinal cord. JAMA LVIII:1489-1490, 1912

18. Tranmer BI, Tucker WS, Bilbao JM: Sleep apnea following percutaneous cervical cordotomy. Can J Neurol Sci 14:262267, 1987

19. Yegul I, Erhan E: Bilateral CT-guided percutaneous cordotomy for cancer pain relief. Clin Radiol 58:886-889, 2003

\section{Disclosures}

The authors report no conflict of interest concerning the materials or methods used in this study or the findings specified in this paper.

\section{Author Contributions}

Conception and design: Aquilina, Thompson. Acquisition of data: all authors. Analysis and interpretation of data: Aquilina, Steel, Kirkman. Drafting the article: Steel. Critically revising the article: Aquilina, Kirkman, Thompson. Reviewed submitted version of manuscript: all authors. Approved the final version of the manuscript on behalf of all authors: Aquilina. Study supervision: Aquilina, Thompson.

\section{Correspondence}

Kristian Aquilina, Department of Neurosurgery, Great Ormond Street Hospital for Children NHS Trust, London WC1N 3JH, United Kingdom. email: kristian.aquilina@gosh.nhs.uk. 